\title{
Quand préoccupations environnementales et enjeux professionnels s'entrecroisent : le cas des entreprises participant aux essais de voitures électriques
}

\author{
Magali Pierre ${ }^{\mathrm{a}}$ \\ EDF R\&D, 1 avenue du Général de Gaulle, BP. 408, 92141 Clamart Cedex, France
}

\begin{abstract}
Résumé. Des expérimentations visant à promouvoir le déploiement du véhicule électrique se sont fait jour dans le cadre de projets démonstrateurs, depuis quelques années. Elles ont en commun de proposer à des salariés d'utiliser ces voitures dans des conditions très similaires au futur proche. Ce texte vise à la fois à mettre en avant les raisons que les différentes parties prenantes - qu'il s'agisse des directions en charge des achats ou du développement durable, des gestionnaires de flotte, des utilisateurs finaux, etc. ont de s'inscrire dans ces expérimentations, et à expliciter la prédominance des enjeux professionnels, qui continuent de déterminer le rapport à cette voiture. En effet, les registres professionnels (enjeux de reconnaissance, d'exemplarité) et personnels se combinent dans l'adoption de cette technologie de mobilité durable, que la préoccupation environnementale ne suffit pas à expliquer.
\end{abstract}

\begin{abstract}
Trials aiming at promoting the spread of the electric vehicle have taken place in the framework of demonstration projects for some years. Most of them propose to employees to use these vehicles in conditions that are very similar to the real emerging market ones. This paper intends to analyze the various reasons why stake-holders, such as Departments in charge of Purchasing or Sustainable Development, Car fleet managers or End-users, take part in these trials. At the same time, it insists on the professional challenges that determine the relationship to this vehicle. Indeed, this sustainable mobility technology is adopted for private and professional reasons rather than because of environmental awareness: for instance, recognition within the company and image of the organization are at stake.
\end{abstract}

\section{Introduction}

Une des voies prises par la mobilité soutenable est celle du véhicule électrique (VE) : autorisant des pollutions moindres sans pour autant remettre en cause le système dominant des modes individuels de transports, le VE se présente en effet comme un compromis atteignable vers une mobilité durable. Le VE semble à priori adapté aux flottes commerciales [1], puisque les trajets quotidiens sont plus routiniers, et que les emplacements de stationnement sont fixes la nuit. En outre, il est rassurant, dans les organisations, que d'autres véhicules thermiques de la flotte restent disponibles, le cas échéant, pour

\footnotetext{
${ }^{a}$ e-mail : magali.pierre@edf.fr
}

This is an Open Access article distributed under the terms of the Creative Commons Attribution License 4.0, which permits unrestricted use, distribution, and reproduction in any medium, provided the original work is properly cited. 
le cas où l'autonomie de la batterie du véhicule électrique ne serait pas suffisante à réaliser un trajet. Et les gains d'image peuvent être mis en avant par les organisations impliquées. Dans le même temps, le VE introduit une contrainte oubliée, liée à l'autonomie limitée de sa batterie. Cette limitation prend un relief particulier pour les organisations et les salariés à la mobilité professionnelle contrainte.

L'objectif de cet article est de décrire les relations à la mobilité électrique de personnes en contexte professionnel. À l'appui de cette description, nous saisirons trois contextes d'enquête correspondant à autant de configurations techniques, mais dont les contextes socio-organisationnels sont très ressemblants (cf. synthèse dans le Tableau 1).

\section{Des expérimentations grandeur nature}

Cet article est établi sur la base d'enquêtes réalisées dans le cadre du suivi de trois expérimentations visant à promouvoir le déploiement du VE et de l'infrastructure de charge apparues dans le cadre de projets démonstrateurs qui ont en commun de proposer à des salariés d'utiliser des VE dans des conditions très similaires aux conditions réelles du marché (prix et durées de location des véhicules, les points de charge sont achetés, les VE sont intégrés au parc automobile de l'entreprise, etc.).

En adhérant à l'expérimentation l'entreprise consent à payer tous les mois au constructeur automobile l'équivalent d'une location longue durée. La plupart du temps, l'établissement achète également un coffret de charge installé sur le site professionnel (sauf enquête $\mathrm{C}$ où le point de charge a parfois été offert par les partenaires industriels); certains établissements achètent en outre un coffret de charge pour un de leurs salariés pour permettre la recharge au domicile.

La facturation de la consommation électrique dépend du lieu de charge ; conséquemment, pour les utilisateurs finaux, l'électricité est payante au domicile ; elle est gratuite à la station service, dans les parkings, sur les stationnements sur voirie publique ${ }^{b}$ et chez l'employeur (car prise en charge par ces derniers et les collectivités territoriales).

Ces expérimentations ont pris place sur des territoires variés : dans l'enquête A, les utilisateurs occupent une région rurale au sein de laquelle se trouve une grande ville (qui concentre l'infrastructure publique de charge $^{\mathrm{c}}$ ) ; l'enquête $\mathrm{B}$ a lieu dans un département composé de villes intermédiaires ; l'enquête C couvre un territoire étendu (4 départements dont deux en Allemagne). Malgré cette hétérogénéité, les populations enquêtées ont en commun de réaliser des trajets de moyenne portée en tissu urbain, périurbain et rural. Les populations des enquêtes $\mathrm{A}$ et $\mathrm{C}$ réalisent majoritairement des trajets radiaux alors que l'enquête $\mathrm{B}$ concerne surtout des trajets reliant une ville moyenne à une autre.

\section{Des contextes socio-organisationnels très similaires}

Les configurations techniques des trois expérimentations sont légèrement différentes (cf. Tableau 1) : le premier cas (A dans le tableau qui suit) est celui du véhicule hybride rechargeable, le deuxième (B) celui de différents VE distribués par un même constructeur automobile, et le troisième $(\mathrm{C})$ concerne des VE de marques différentes, se chargeant sur des points de charge dissemblables.

En revanche, les contextes socio-organisationnels des trois terrains d'enquête sont quasi identiques. Dans les trois cas, il s'agit de diffuser des véhicules novateurs au sein d'établissements publics et privés de toutes tailles (de la PME au grand groupe industriel en passant par des collectivités locales diverses). Il a été laissé à la charge des établissements concernés d'allouer les VE sous les formes qu'elles souhaitaient (modalités d'attribution, personnes désignées ou appel à volontaires, etc.). Les

\footnotetext{
${ }^{\mathrm{b}}$ En voirie comme en parkings concédés, le stationnement est généralement payant, comme pour les voitures conventionnelles.

c Nous n'évoquerons pas les usages de cette infrastructure publique de charge (voirie, parkings souterrains, centres commerciaux, stations-service) dans la présente communication. Nous mettrons donc de côté les nombreux enjeux territoriaux de ce déploiement de véhicules électriques.
} 
actifs, désignés ou volontaires, se sont engagés à prendre en charge et utiliser les véhicules sur une période longue.

Les VE s'insèrent dans des flottes de taille importante. Il s'agit souvent de grandes entreprises ou établissements publics avec des flottes conséquentes, pouvant aller de quelques dizaines à plusieurs centaines de véhicules. Dans ces établissements, les flottes de voitures thermiques sont acquises la plupart du temps et/ou louées. Dans ces flottes, la plupart du temps une partie des véhicules sont en pool, une autre est attribuée à des services et le reste est attribué à des personnes. C'est aussi le cas des VE.

Plusieurs familles de métiers émergent parmi les personnes interrogées : des cadres intermédiaires s'occupant de domaines tels la logistique et les moyens généraux (responsables bâtiment dans un établissement public, responsables du matériel dans une société de BTP, etc.), ou encore l'action sociale et la gestion (contrôleur de gestion, directeurs de magasin, etc.); des ingénieurs et quelques professions indépendantes (artisans); et enfin, des techniciens dont les déplacements ont été repérés comme cadrant parfaitement avec les capacités du VE (mécaniciens, chargés de sécurité ou de maintenance, service courrier, électriciens, etc.).

Deux types de statuts professionnels sont ainsi représentés : des employés (maintenance, sécurité, etc.) et des responsables (services généraux, développement durable, achats, etc.). Les secteurs d'emploi sont l'énergie, l'action sociale, le BTP, l'action municipale, etc.

\section{Méthodologie}

Les enquêtes relatées ont été menées entre 2010 et 2012. Le recrutement a été fait sur la base des fichiers clients, fournis par les partenaires industriels de l'expérimentation. Les entretiens auprès des expérimentateurs ont été réalisés sur les lieux des pratiques, autrement dit sur les sites professionnels des personnes interrogées ou à leur domicile. L'enquête B a été réalisée conjointement avec le LVMT ${ }^{\mathrm{d}}$. Nous analyserons les pratiques énergétiques des entreprises à travers les actions en faveur du VE et montrerons comment les projets de déploiement du VE sont perçus au sein des organisations utilisatrices. Nous nous demanderons enfin de quelle manière le VE est un objet qui reconfigure les organisations et les mobilités des personnes.

Lorsque le particulier consomme de l'énergie il cumule les rôles car il contractualise avec l'énergéticien, consomme et acquitte le paiement des factures. Il est également l'acheteur, le gestionnaire et l'utilisateur d'une voiture. Le salarié lui est l'utilisateur mais pas le payeur (sauf dans le cas où il utilise le VE chez lui et paie alors son électricité). Il n'a donc pas d'intérêt économique à réduire la facture. En outre, le salarié n'est pas un utilisateur comme les autres : il n'est pas en position de consommateur mais d'employé (allégeance à l'entreprise, efficacité nécessaire, besoin de reconnaissance) et de testeur (voire de « cobaye », un registre plus personnel et identitaire).

Cette analyse considère les expérimentateurs à la fois comme des utilisateurs d'un dispositif technique, des porteurs (ou détracteurs) de normes environnementales inscrites dans les objets techniques et dans les modalités de leur diffusion, et des employés au sein d'organisations. Elle se situe ainsi à la croisée de la sociologie des usages, de la sociologie des sciences et techniques et de la sociologie de l'innovation et des organisations.

\footnotetext{
d Plus précisément, cette enquête $\mathrm{B}$, dont une partie des résultats est restituée ici, a été réalisée conjointement par Magali Pierre (EDF R\&D) et une équipe de l'université Paris-Est Marne-la-Vallée (LVMT- Laboratoire Ville, Mobilité, Transport) comprenant Marie-Hélène Massot, Anne Jarrigeon, Benjamin Pradel et Marianne Thébert. Le rapport d'étude de cette enquête commanditée par EDF R\&D et Renault s'intitule Image, Usages et territorialisation d'un système de mobilité électrique : analyse de l'expérimentation SAVE.
} 


\section{SHS Web of Conferences}

Tableau 1. Résumé des principales caractéristiques des trois enquêtes réalisées.

\begin{tabular}{|c|c|c|c|}
\hline & Enquête A & Enquête B & Enquête C \\
\hline $\begin{array}{l}\text { Types de VE } \\
\text { (pur électrique ou hybride } \\
\text { rechargeable, modèle, } \\
\text { etc.) }\end{array}$ & $\begin{array}{l}\text { Véhicule hybride } \\
\text { rechargeable (VHR). Des } \\
\text { berlines }\end{array}$ & $\begin{array}{l}\text { «full » électrique (VE). } \\
\text { Des VUL et berlines d'une } \\
\text { même marque }\end{array}$ & $\begin{array}{l}\text { «full » électrique (VE). } \\
\text { Tous types de VE } \\
\text { (marques diverses, tailles } \\
\text { diverses, etc.) }\end{array}$ \\
\hline Nombre de VE déployés & $\begin{array}{l}80 \text { véhicules } \\
\text { (expérimentation) }\end{array}$ & $\begin{array}{l}53 \text { véhicules } \\
\text { (expérimentation) }\end{array}$ & $\begin{array}{l}\text { Des centaines de } \\
\text { véhicules dont environ } \\
80 \text { en entreprises } \\
\text { (déploiement de masse) }\end{array}$ \\
\hline $\begin{array}{l}\text { Nombre d'utilisateurs } \\
\text { (dont utilisateurs de VE } \\
\text { partagés) }\end{array}$ & Environ 150 & $\begin{array}{l}85 \text { badges distribués, donc } \\
\text { sans doute entre } 100 \text { et } \\
150 \text { utilisateurs }\end{array}$ & $\begin{array}{l}\text { Plusieurs centaines. } 118 \\
\text { badges distribués (accès } \\
\text { à l'infrastructure } \\
\text { publique de charge) }\end{array}$ \\
\hline $\begin{array}{l}\text { Nombre d'utilisateurs } \\
\text { interrogés }\end{array}$ & $\begin{array}{l}\text { Environ } 30 \text { à } 4 \text { reprises } \\
\text { (entre } 25 \text { et } 35 \text { à chaque } \\
\text { phase d'enquête), dont } \\
\text { environ } 10 \text { gestionnaires } \\
\text { de flotte }\end{array}$ & $\begin{array}{l}30 \text { utilisateurs, } \\
13 \text { gestionnaires de VE } \\
\text { (utilisant } \\
\text { occasionnellement le VE) }\end{array}$ & $\begin{array}{l}40 \text { dont } 25 \text { en contexte } \\
\text { professionnel (le reste } \\
\text { sont des particuliers - } \\
\text { hors enquête) ; parmi ces } \\
25 \text { personnes, une } \\
\text { dizaine sont des } \\
\text { gestionnaires de flotte }\end{array}$ \\
\hline $\begin{array}{l}\text { Utilisateurs } \\
\text { interrogés réalisant aussi } \\
\text { des trajets domicile-travail } \\
\text { et/ou privés avec le VE }\end{array}$ & $\begin{array}{l}\text { Sur chaque phase, environ } \\
15 \text { à } 20 \text { personnes (= ont } \\
\text { des coffrets domestiques) } \\
\text { parmi eux, une dizaine } \\
\text { pour des motifs privés } \\
\text { (voiture de fonction) }\end{array}$ & $\begin{array}{l}19 \text { font des trajets } \\
\text { domicile-travail. } 8 \text { ont un } \\
\text { coffret domestique } \\
\text { (charges intermédiaires) } \\
=\text { motifs privés }\end{array}$ & $\begin{array}{l}\text { Une bonne moitié charge } \\
\text { occasionnellement chez } \\
\text { soi. VE ne nécessitant } \\
\text { pas de coffrets de charge }\end{array}$ \\
\hline $\begin{array}{l}\text { Durée de } \\
\text { l'expérimentation }\end{array}$ & 3 ans & 1,5 an & \\
\hline
\end{tabular}

\section{Le VE, un objet polymorphe pour les organisations}

Toute participation au test des véhicules nécessite un investissement humain et financier de la part des partenaires. Pour quelles raisons les expérimentateurs le concèdent-ils ? Les raisons liées à la préservation de l'environnement, transversales parmi les personnes interrogées, sont nécessaires mais pas suffisantes pour rendre compte de l'engagement dans l'expérimentation. Comment le système sociotechnique qui comprend VE+ l'infrastructure de charge et la consommation d'énergie est-il considéré par les membres de l'organisation - notamment les décideurs, les gestionnaires de flotte et les salariés - alors que leurs intérêts différents ?

\section{Un consensus sur le fait que le VE est une voiture plus « propre »}

Les qualités du véhicule électrique (VE) mentionnées couvrent le champ environnemental : silence de conduite, réduction des émissions de $\mathrm{CO} 2$ et des pollutions locales, etc. À quoi s'ajoutent la moindre dépendance aux énergies fossiles et le plaisir de ne plus avoir à aller à la station service, cumulés au fait que le coût en énergie est moindre. Dans les organisations, le VE est renvoyé à des enjeux énergétiques et environnementaux par l'ensemble des personnes interrogées ${ }^{\mathrm{e}}$, même si cet aspect recouvre des éléments variés. Ces bénéfices escomptés sont à l'origine de la participation

\footnotetext{
e Notons que certaines mentionnent également, en parallèle, les risques environnementaux que représentent le recyclage des batteries et les externalités liées à leur fabrication. Quelques-uns rappellent également le besoin en énergie électrique et son mode de production (majoritairement issu du nucléaire).
} 
des établissements concernés. De plus, l'expérimentation déploie un dispositif novateur dont l'enjeu est important pour les porteurs du projet comme pour les expérimentateurs, et fonctionne comme un dispositif d'engagement, d'enrôlement.

\section{Différentes populations, différentes perceptions}

Les raisons de l'implication des différentes entités concernées - qu'il s'agisse des directions en charge des achats ou du développement durable, des gestionnaires de flotte, des utilisateurs finaux, etc. varient. En effet, si les membres de l'organisation connaissent des contraintes propres dans lesquelles le VE et son système sociotechnique doivent s'insérer, cette multiplicité de contraintes entraine une perception différente de l'objet. Les populations qui constituent les organisations sont donc autant de publics appréhendant différemment les expérimentations qui se présentent à eux. Précisons pour chacun ${ }^{\mathrm{f}}$ les contraintes, les ressources et les zones d'incertitude.

\section{Pour le sommet stratégique}

Pour les entreprises et les institutions qui participent (directeurs du développement durable, chefs de PME, directeurs des achats, etc.), les raisons de participer à un projet de mobilité durable sont nombreuses :

- Effectuer un test technique et d'acceptabilité dans l'optique d'équiper sa flotte de véhicules d'entreprises en VE pour créer un précédent dans l'entreprise.

- Innover et se montrer pionnier dans l'usage d'une voiture perçue comme un symbole du progrès technique.

- Se saisir d'une opportunité : nombre de participants sont directement intéressés par les résultats de l'expérimentation car ils gravitent autour des domaines de l'énergie ou du VE.

D'autres gains sont ponctuellement envisagés, comme :

- Les économies d'énergie abordées sous l'angle du gain financier (économies escomptées en termes de consommation d'énergie).

- Remporter des marchés publics : une flotte plus « durable » est, pour certains secteurs, une condition d'obtention de marchés publics.

Pour les décideurs, le coût ne semble pas être la variable décisive car cela permet de montrer que l'établissement consent à des efforts en faveur de la mobilité durable qui renvoie à un impératif de RSE (responsabilité sociétale des entreprises). En outre, des groupements d'achat ainsi que les subventions et remises (de l'ADEME, des collectivités locales, etc.) ont fait baisser ces coûts d'investissement.

\section{Pour les collectivités locales}

Les collectivités locales testent les VE et installent des points de charge sur la voie publique. L'engagement des collectivités locales dans l'expérimentation est donc motivé par la mission de développement économique du territoire qui est la leur. C'est ce soutien à la filière automobile, et non pas des enjeux de mise à disposition d'un service public de points de charge, qui justifie ainsi l'aide au déploiement de points de charge accessibles sur les lieux publics. Par ailleurs, les communes impliquées ont tendance à justifier leur engagement par l'opportunité de tester des véhicules électriques - l'autonomie des VE suffit généralement aux besoins des villes pour les déplacements locaux - et par le

\footnotetext{
${ }^{\mathrm{f}}$ Nous segmentons les membres de l'organisation en nous en remettant à la typologie de Mintzberg [3] qui distingue le sommet stratégique, la technostructure, la ligne hiérarchique, le centre opérationnel et la logistique.
} 
désir de montrer aux administrés l'engagement en faveur du développement durable. Les établissements investissant massivement sont les mairies pour honorer les chartes sur lesquelles elles se sont engagées.

\section{Pour la hiérarchie intermédiaire}

Pour les entreprises, la mobilité des salariés est une nécessité pour assurer le fonctionnement de l'organisation et une source forte de risque, car il est difficile de maîtriser l'incertitude que représente un employé hors du site professionnel. Le découpage de l'activité en sous-tâches est alors une des manières de retrouver une maîtrise de cette zone d'incertitude [4] : les moments-lieux-personnes responsables de la préparation de l'activité, du trajet, de l'intervention, de la réalisation et de son contrôle sont ainsi dissociés. Le VE rajoute à cette incertitude car il fournit une occasion de perdre du temps (le temps nécessaire à la recharge, à sa vérification, à la prise en main du véhicule, etc.); mais en même temps il réduit une partie de cette incertitude, puisque sa gestion rapprochée occasionne un contrôle du moment du trajet et redonne donc du pouvoir au manager. En outre, pour la hiérarchie intermédiaire, le VE est considéré comme un outil de valorisation du personnel ainsi que le prouvent les concours (concours de vente, concours de production) à l'origine de la sélection des usagers des véhicules.

Pour le salarié, le VE est à positionner en termes d'efficacité : il s'agit de ne pas perdre de temps pour réaliser sa tâche. De ce point de vue, l'innovation et la valorisation qu'il apporte sont gommées par la contrainte qu'il représente.

\section{Le cas des gestionnaires (services Logistique et Moyens généraux)}

Pour les gestionnaires de flotte, le VE est à la fois une contrainte et une innovation.

\section{Gestionnaire de parc automobile, un métier complexe}

Le gestionnaire de la flotte de véhicule est à la fois le garant des coûts de déplacements et un chef d'équipe (ces dernières se composant de mécaniciens, techniciens, etc.), deux aspects de son métier qui permettent de comprendre son positionnement face au VE. En effet, le VE permet de réduire les coûts de maintenance et les coûts de carburant, mais les gestionnaires de flotte considèrent que les gains en coûts d'exploitation ne compensent pas la perte en coût d'achat (les VE sont réputés chers) sans avoir toutefois réalisé de bilan ni établi de comparaison avec un VT alors qu'en termes de risque économique le leasing ${ }^{\mathrm{g}}$ est très bien accepté. La difficulté de calcul du bilan tient, d'une part à la valeur de revente des VE encore inconnue et, d'autre part, aux frais de carburant imputés aux services Parc Auto alors que les frais d'électricité sont imputés aux services maintenance ou patrimoine immobilier.

Le VE est également une cause de réduction du parc, du fait de son coût d'acquisition : un $\mathrm{VE}=2 \mathrm{VT}$ (enquête B notamment), autrement dit investir dans le VE peut empêcher d'autres achats. De plus, les gestionnaires de flotte sont souvent les responsables des garages. L'arrivée du VE oblige à une recomposition des métiers de mécanique (voire leur disparition). Le VE est donc une cause de réduction du parc automobile et des équipes.

\section{Un VE complexe à gérer}

Le VE est de plus un véhicule complexe à gérer car il nécessite de :

\footnotetext{
g La LLD (location de longue durée), qui peut éventuellement inclure des services comme l'entretien, l'assurance, la gestion du carburant et des pneumatiques, se répand de plus en plus dans les flottes des grands groupes notamment car elle permet de préserver des capacités de financement et de prévoir les dépenses [5]. De plus, la location de la batterie paraît généralement assez intéressante aux gestionnaires car elle permet : 1/ de baisser le coût d'achat et 2/ de lisser l'aléa de la panne de batterie.
} 
- Former les utilisateurs novices : les gestionnaires de flotte ont bien souvent la charge de former les utilisateurs à la conduite du VE et à sa recharge. Il s'agit également de motiver « ceux qui ne veulent pas lâcher leur thermique ».

- Prendre en compte les capacités limitées de rayonnement : cela implique d'évaluer systématiquement les distances que l'utilisateur va parcourir. Le court rayon d'action limite les possibilités d'affectation.

Le VE étant doté d'une autonomie en mode électrique considérée comme faible (au réel, de 100 à $160 \mathrm{~km}$ selon les types de VE) est privilégié pour les trajets suivants correspondant à des courtes distances ou à des trajets très routiniers :

- Petites courses

- Trajets entre sites, par exemple pour la distribution du courrier

- Démonstration : trajets visant à montrer le véhicule

- Trajets domicile-travail.

Ainsi, l'attribution du véhicule impose de réfléchir en amont à son usage et donc à établir des diagnostics contrairement aux VT et impose une contrainte supplémentaire aux gestionnaires de flotte. Les services prioritaires sont les coursiers, le garage, la petite maintenance locale, le suivi de chantiers, le transport de personnel entre deux sites relativement proches, les releveurs, etc. Le VE peut être alloué de deux manières principales :

- Affectation simple en tant que voiture de fonction ou de service attribuée à une personne (ou à deux, qui connaissent leurs plannings mutuels), car cela reporte cette gestion de l'autonomie sur l'utilisateur.

- En tant que véhicule du pool, partagé entre de nombreux salariés ; cela nécessite la participation du gestionnaire pour réattribuer à chaque fois le VE en fonction des usages pressentis. L'utilisation partagée est parfois vue comme une manière de ne pas empêcher des usages puisque les moments de recharge correspondent aux moments de rotation.

Cette anticipation des trajets place le gestionnaire dans une posture réflexive qui a conduit à revaloriser la fonction d'attribution des véhicules, ce que certains gestionnaires apprécient quand d'autres refusent (« on n'est pas des loueurs »).

\section{Gérer les emplacements de stationnement (protection vol, sécurité électrique, anticiper le} temps de recharge nécessaire)

La mise à disposition du VE s'accompagne de la nécessité de gérer également les lieux de stationnement, afin que le VE puisse être rechargé dans de bonnes conditions. Ainsi, dans la « charte » composée par quelques gestionnaires de VE, il est exigé le respect de conditions favorables de stationnement. Si cette charge est aisée dans le cas de VE attribués à une seule personne, comment les entreprises concilientelles temps de charge et utilisation partagée du VE ? Dans les flottes d'entreprises, les trajets sont en boucle (ce qui n'est pas toujours le cas dans les sociétés d'auto-partage), ce qui simplifie la recharge. Par ailleurs pour les VE partagés circule souvent une consigne de charge systématique du VE lors du retour sur site.

\section{Une solution : l'assouplissement des conditions d'utilisation}

Il est fréquent que les personnes en charge de l'insertion du VE dans les établissements assouplissent les règles de mise à disposition du $\mathrm{VE}$ afin de permettre la recharge sur des points secondaires (aux domiciles, sur des sites publics, etc.). Il s'agit avant tout d'une manière de convaincre les utilisateurs, à priori réticents, de passer le cap et de sécuriser l'utilisateur (psychologiquement, mais 
aussi objectivement) par rapport à l'autonomie. Mais cela témoigne également de l'adaptabilité forte des personnes en charge du VE, ayant compris que la réussite de ce test passait par une souplesse d'utilisation à laquelle la gestion des flottes de voitures conventionnelles n'est pas coutumière.

\section{Le VE, un objet qui reconfigure les mobilités des salariés}

Nous nous concentrons ici plus particulièrement sur le sens que revêt l'implication dans ces expérimentations pour les utilisateurs de VE en soulignant l'importance des enjeux professionnels, qui déterminent le rapport à cette voiture et se cristallisent autour de la gestion de l'autonomie du véhicule. Des enjeux statutaires et des éléments liés à la reconnaissance sociale (managériale, et exemplarité) tout au long de la «vie » de l'objet [6], c'est-à-dire aux différentes étapes de l'attribution-usage-chargerestitution du VE se manifestent.

Le VE représente une contrainte dans le contexte des activités professionnelles : brancher demande du temps (coût d'apprentissage, réalisation quotidienne), voire de l'argent (facture si charge à domicile); et planifier ses trajets rajoute une zone d'incertitude quant au délai du déplacement. L'autonomie limitée représente également une contrainte pour les utilisateurs.

Si la plupart des utilisateurs se font un point d'honneur à préserver leurs routines de déplacements, ce qui est d'autant plus simple que ceux-ci se concentrent autour du lieu de travail et dans des périmètres restreints, pour tous le VE requiert une anticipation des trajets qui place l'utilisateur et le gestionnaire dans une posture réflexive.

\section{L'autonomie limitée, une contrainte qui oblige à une mobilité réflexive}

\section{Une vraie rupture}

Le VE donne lieu à une vision positive de la conduite automobile et suscite un rapport sensible renouvelé à l'environnement et au territoire quotidien. En effet, il ouvre une nouvelle ère dans l'expérience automobile, en particulier par sa puissance et son silence, puissants leviers d'un rapport inédit à l'environnement automobile. Le véhicule électrique et « son silence », par-delà les risques qu'il présente en termes de sécurité routière, révèle un désir d'apaisement des interactions automobiles, qui entre de façon primordiale dans le renouvellement du rapport sensible à l'environnement de conduite. «C'est la zénitude au volant », déclare un des utilisateurs. L'imaginaire de l'automobile vrombissante associée à une conduite en tension avec les autres véhicules est ainsi contrecarré par un imaginaire rassérénant. La dynamique de changement initiée par un dispositif visant à promouvoir une mobilité plus durable [7] est une réussite dans la mesure où pour les usagers, le VE est une véritable voiture, c'est-à-dire à la fois une voiture normale et une voiture novatrice.

\section{Éco-conduire}

Les utilisateurs composent avec une définition ambiguë de l'autonomie, qui fait l'objet d'annonces par les constructeurs automobiles, mais aussi d'évaluations subjectives et d'attentes symboliques fortes. La perception de l'autonomie va dépendre de la familiarité avec le dispositif. Au début de l'apprentissage, on se demande si les données fournies sont fiables ; une fois l'apprentissage passé, on apprend à faire confiance (non pas à l'autonomie théorique déclarée, mais à l'autonomie réelle que l'on a expérimenté), voire on revient au tableau de bord fort de son expertise, pour vérifier ce que l'on sait désormais ${ }^{\text {h. Bien }}$

\footnotetext{
h Notre dispositif d'enquête a permis de saisir cet effet d'apprentissage, puisque les entretiens ont eu lieu dans une période où la phase de découverte est généralement passée depuis plusieurs mois; en entretiens, nous avons pu noter que les utilisateurs ont du recul sur l'évolution de leurs pratiques et représentations.
} 
entendu, cet apprentissage est plus problématique dans le cas d'utilisateurs occasionnels, qui eux le subissent à chaque utilisation du VE.

La consommation d'énergie est au cœur d'un dispositif particulièrement incitatif disposé sur le tableau de bord, qui pousse les conducteurs à s'instituer spontanément en éco-conducteur : afin de conserver le maximum de capacité de batterie, les utilisateurs réduisent leur vitesse, freinent pour récupérer de l'énergie, ou encore recherchent le «point d'équilibre » qui permet de rouler sans consommer d'électricité.

\section{Anticiper et sélectionner ses propres trajets : privilégier les routes nationales}

Le conducteur apprend ainsi à charger son véhicule, gérer l'autonomie, interpréter les informations du tableau de bord, tout en y intégrant les aménités du territoire (réseau viaire, stationnement, bornes), les variables environnementales, les caractéristiques des trajets à effectuer et les besoins professionnels. L'usage du véhicule électrique s'appuie ainsi sur des exercices nécessaires d'anticipation et de programmation (agendas surlignés, vérification de l'emploi du temps la veille du déplacement, etc.). Les conducteurs apprennent alors à identifier les situations où l'autonomie ne serait pas suffisante. Avec le temps, cette anticipation devient elle aussi un automatisme incorporé aux routines ; c'est le cas des trajets comme des stratégies de charge, qui deviennent progressivement de simples habitudes.

\section{Apprendre du véhicule}

La problématique de l'autonomie se réduit à mesure de l'expérience de conduite : connaissance technique du VE, utilisation ajustée des bornes de charge. Par exemple, on différenciera la charge systématique des personnes partageant le VE de la charge raisonnée de ceux qui en disposent à titre personnel, c'est-à-dire qui maitrisent parfaitement le planning de sortie du VE. En effet, la charge raisonnée a lieu après considération du besoin pour le trajet suivant. Elle a nécessité une phase d'apprentissage pour connaître ses propres comportements de déplacements ${ }^{\mathrm{i}}$, autrement dit pour les analyser au regard des performances du VE (en termes d'autonomie).

En somme, les conducteurs de véhicules électriques s'adaptent à cette autonomie limitée par une série d'ajustements qui prennent la forme d'une nouvelle organisation du quotidien (la conduite économe, la routinisation des déplacements, l'identification des situations limites, le recours exceptionnel à un véhicule secondaire pour les longs trajets, l'anticipation des moments d'usage du véhicule ou encore la prévision des lieux de stationnement), inscrivant les déplacements dans une dynamique de réflexivité. De même, la voiture électrique nécessite une gestion particulière de la charge et des stratégies adaptées de recharge, afin d'intégrer ces nouveaux gestes aux activités quotidiennes.

\section{Des postures différenciées}

Tout le monde ne s'adapte pas de la même manière aux changements comportementaux appelés par le système technique : ce degré d'implication dépend des valeurs, de la capacité décisionnaire, du métier et du portage du projet. Ainsi, les adaptations en termes de réflexivité et de trajets sont sans doute plus importantes dans les enquêtes B et C (il s'agit de VE) que dans l'enquête A (VHR). Mais le sentiment d'une nouvelle ère dans la mobilité tient également souvent au standing du véhicule, comme le montrent les trois enquêtes.

Bien que la plupart des participants à l'expérimentation soient au fait des avantages des véhicules à traction électrique, leur implication ne va pas de soi pour autant. Bien entendu, s'impliquer dans le

\footnotetext{
${ }^{\text {i }}$ Il arrive que ceux qui partagent le VE en petits groupes le chargent cependant de manière raisonnée, car au bout de quelques mois, ils connaissent leurs trajets et le comportement du véhicule et savent qu'elle est la meilleure fréquence de recharge.
} 


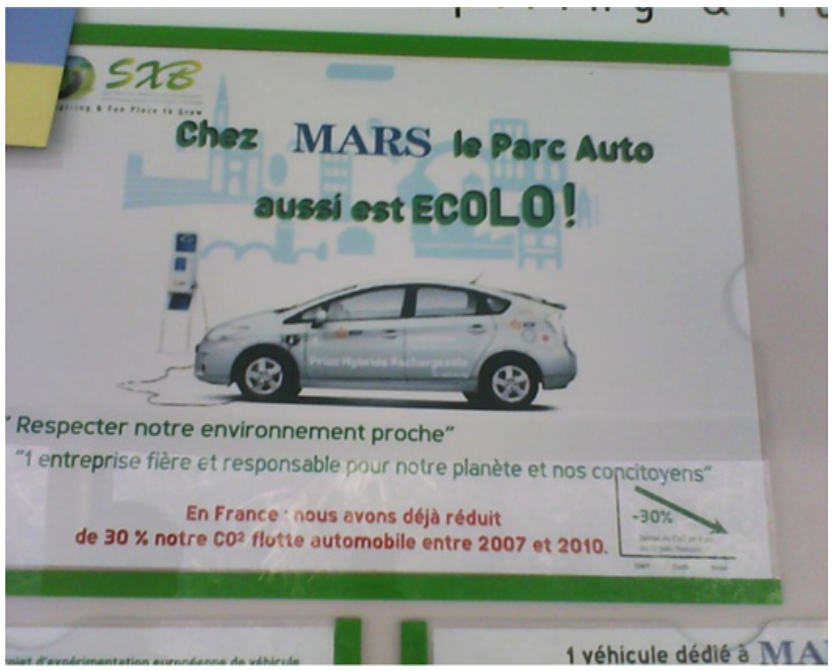

Photo 1. Une communication d'entreprise enthousiaste au sujet du VHR : l'enjeu communicationnel.

projet nécessite que l'on ait pris connaissance du fonctionnement du dispositif, en suivant les réunions à ce sujet, en lisant la documentation fournie, en remplissant son dossier de candidature, etc. (présentation en interne, connaissance des enjeux et des délais de livraison du dispositif, relations antérieures avec les partenaires, etc.). Mais outre ces connaissances préalables, l'origine de l'implication dans le projet relève de valeurs environnementales, de la capacité de décision du participant, du métier ou encore les conditions de déploiement des VE.

\section{Le sentiment d'urgence des enjeux environnementaux liés à la mobilité individuelle}

L'implication dans l'expérimentation suit des logiques différentes selon qu'on est ou non persuadé de l'importance des enjeux du dérèglement climatique et de la pollution locale, de la responsabilité de l'homme sur les émissions de gaz à effet de serre, ou encore de l'intérêt des véhicules à traction électrique pour résoudre ces problèmes. Les plus positifs des utilisateurs permanents trouvent le véhicule hybride rechargeable très performant, et sont vraiment séduits par le fait de pouvoir rouler en mode électrique. Ils se font ainsi les apôtres de cette technologie, n'hésitant pas à transporter des collaborateurs. Cette expérimentation rencontre leurs convictions sur la nécessité de ne pas gaspiller ou encore de se situer sur un modèle durable de développement économique. Ces utilisateurs sont dans une logique « communicationnelle » qui consiste à diffuser la «bonne parole » dans l'entreprise en allant au devant des échanges avec les collaborateurs au sujet du VHR.

Les motivations personnelles rencontrent les efforts consentis et contiennent une implication « identitaire » (cf. plus loin). En effet, ces « utilisateurs réguliers » enthousiastes ont le sentiment d'une implication personnelle, notamment liée aux efforts qu'ils font éventuellement pour participer à l'expérimentation.

\section{La capacité décisionnaire du bénéficiaire}

Quel est le niveau et la nature des ressources dont les participants bénéficient pour faire face à cette innovation? Certains utilisateurs sont des cadres dirigeants ou des chefs d'entreprise à l'initiative de la participation de leur établissement à l'expérimentation. D'autres ont pu être rattachés au projet par 
la voie hiérarchique, et ces aspects prennent alors le pas sur la perception du dispositif. Au sein des départements élus, le choix des utilisateurs finaux (qui aura le droit d'usage des VHR) reste en général un geste managérial top/down. En effet, il ne s'agit pas de n'importe quelle voiture : ce n'est pas une voiture « de technicien » mais une « berline de luxe».

\section{Les métiers}

Les métiers fournissent une grille de lecture pour comprendre la réception qui est faite d'une innovation telle que le véhicule électrique. Si certains professionnels apprécient la mise en visibilité offerte par le véhicule - et notamment ceux qui ont à porter la politique de développement durable de leur organisation d'appartenance, d'autres peuvent se sentir menacés par l'arrivée d'une voiture qui, en modifiant les rapports de force, risque de leur faire ombrage comme les mécaniciens, priés de ne pas ouvrir le capot du véhicule testé, les gestionnaires de flottes pour qui la durée de charge constitue une contrainte pour la gestion du parc, ou encore aux chauffeurs qui voient dans les véhicules électriques le déclin de leur métier, car c'est une voiture bien souvent conduite directement par l'utilisateur (à des fins de démonstration), autrement dit sans chauffeur.

\section{Les modalités de mise à disposition des véhicules}

Les modalités de mise à disposition, c'est-à-dire des ressources et contraintes fournies à chacun pour se servir de ce véhicule - et notamment le fait de pouvoir charger le VE chez soi ou non [8] comptent parmi les conditions de déploiement des véhicules. La fréquente autorisation de rentrer chez soi avec le VE, voire de le recharger chez soi sont appréciées. Ainsi les conditions expérimentales prennent-elles les avantages à la fois des voitures de fonction (qu'on peut rapporter chez soi) et des voitures partagées (que tout le monde peut utiliser), des conditions à priori propices à la réussite du test ${ }^{j}$ (enquête C).

\section{Des registres d'implication prenant des tonalités diverses}

La dimension professionnelle est très présente dans l'implication. Prenons deux exemples retrouvés de façon récurrente dans les trois enquêtes : celui de la logique identitaire et celui de la logique méritoire, qui renvoient toutes deux à des enjeux professionnels et personnels (enquête A).

\section{Logique identitaire}

La logique identitaire invoque une notion d'exemplarité environnementale en situant ses adeptes sur une sphère personnelle, puisqu'elle consiste à être en concordance avec un engagement personnel ou familial. La préoccupation environnementale peut en effet être vécue sur un mode personnel, en accord avec des valeurs profondes. Cette implication conforte ainsi des motivations personnelles qui font supporter les efforts et permettent d'accepter de rétrograder de standing de voiture, de se charger le plus possible, etc. Ces salariés apprécient le soutien de leur famille et notamment de leurs enfants dans ce projet. Cela est également parfois le cas de familles au départ assez réticentes qui ont été séduites par le niveau de confort et le silence du véhicule.

L'implication identitaire peut même aller jusqu'au souhait d'acheter un VE ou un véhicule hybride rechargeable (VHR) à titre personnel, sous réserve de considérations de coûts. Le VE devient alors une manière de faire la preuve d'un engagement de l'entreprise et de ses membres (manager versus exécutants) sur les questions d'économies d'énergie.

j Même si dans les faits, il peut y avoir un danger avec les installations électriques dont la conformité électrique n'a pas été vérifiée. 


\section{SHS Web of Conferences}

\section{Logique méritoire ou opportuniste}

Les individus se situant dans une logique méritoire voient dans l'expérimentation le moyen d'accéder à un véhicule d'entreprise haut de gamme et de recueillir ainsi un signe de reconnaissance professionnelle. En effet, une voiture de société, notamment quand elle est attribuée à titre personnel, est une récompense managériale d'importance que certains refusent. L'expérimentation a par exemple pu ainsi accélérer une promotion. Certains voient également dans ce dispositif la possibilité de passer d'une voiture de service ou personnelle à une voiture de fonction - et donc de se débarrasser d'un véhicule familial. Ces logiques dépendent de la manière dont les utilisateurs ont été impliqués dans l'expérimentation, c'est-à-dire selon qu'elle est passée par la voie hiérarchique, sur un mode horizontal ou sous la forme d'une promotion individuelle. Les facteurs qui priment sont alors le métier (plutôt peu qualifié) et le statut du véhicule (voiture de service).

La question de l'acquittement des frais de consommation d'électricité à domicile rend cette logique méritoire encore plus saillante. En effet, en vertu de cette logique, l'échange entre l'entreprise et le salarié doit avoir un solde neutre (don/contre-don $=0$ ). Or le VE, lorsqu'il est utilisé à domicile, questionne le contrat selon lequel l'entreprise n'a pas de dette par rapport au salarié car ce dernier paie pour certaines consommations de son véhicule professionnel. Quand cette mise à disposition est gracieuse (dans le cas de voitures de service, pour qui la possibilité de rentrer chez soi avec la voiture professionnelle est une tolérance), ce coût ne pose en général pas de problème. Pour le dire vite, puisque l'entreprise est libérale, le salarié l'est également. Mais si un rapport conflictuel entre le salarié et sa hiérarchie perturbe cette équation, le premier pourra refuser de charger chez lui.

Quand cette mise à disposition est non plus gracieuse mais contractuelle (dans le cas de voitures de fonction), le salarié juge l'effort supplémentaire demandé en fonction de son rapport à l'organisation : il peut le trouver intolérable ou le consentir volontiers et puise alors sur un registre personnel la preuve de sa bonne volonté par rapport à l'organisation en acceptant de rétrograder de standing de voiture, de se charger le plus possible, etc.

\section{Conclusion}

Le déploiement de VE dans le secteur des flottes automobiles est un des facteurs soutenant son essor [9]. Pour autant, les enjeux soulevés par ce déploiement ne se situent pas toujours sur un registre environnemental mais bien souvent professionnel. Les salariés pris comme des utilisateurs manifestent des logiques diverses d'implication, en fonction non seulement de leurs valeurs personnelles mais également sur un registre professionnel. Ainsi, les logiques d'implication dans l'expérimentation sont un marqueur des enjeux professionnels qui se jouent à l'échelle des salariés : enjeux de reconnaissance, d'exemplarité, etc. Par ailleurs, elles révèlent les contraintes propres aux organisations : contraintes de temps, d'efficacité, de réussite, de loyauté par rapport à un corps de métier, etc. Les deux logiques (identitaire et méritoire) que nous avons prises pour illustration montrent comment se combinent les registres professionnels et personnels dans l'adoption d'une technologie de mobilité durable.

Pour comprendre le rapport des professionnels à la mobilité électrique, il nous faut considérer, outre le système technique, l'importance des conditions de déploiement. Deux facteurs sont discriminants : les modalités de mise à disposition, le fait de pouvoir rentrer chez soi avec le VE (voire de pouvoir le charger chez soi). Nous noterons donc l'importance de l'assouplissement des règles d'utilisation, tant du point de vue de la gestion que de l'utilisation : pouvoir rentrer chez soi avec le VE est un changement organisationnel certes délicat à initier, mais nécessaire du point de vue de l'utilisateur. Ce besoin souligne la reconfiguration de la mobilité à l'œuvre dans les milieux professionnels, faisant intervenir de manière entremêlée les déplacements et le stationnaire, et les aspects privés et professionnels. 
Ainsi, le VE à la fois est un marqueur de la diversité des enjeux qui se jouent au sein des organisations, et il reconfigure la mobilité des salariés, en réactivant l'importance du stationnement et des trajets domicile-travail.

\section{Références}

[1] J. Globisch, U. Schneider, E. Dütschke, "Acceptance of electric vehicles by commercial users in the electric mobility pilot regions in Germany", ECEEE Summer Study Proceedings 2013 (2013)

[2] R. Ozaki, K. Sevastyanova, "Going hybrid: an analysis of consumer purchase motivations", Energy Policy, 39, pp. 2217-2227 (2011)

[3] H. Mintzberg, Structure et dynamique des organisations, Edition d'Organisation (1982)

[4] A. Largier, " La maitrise des temps de l'activité en situation de mobilité : un enjeu organisationnel », in C. Caron, G. Gaglio, L'organisation à l'épreuve. Autour du temps, de la sociabilité, de la rationalité et du métier, PUR, Rennes, pp. 25-40 (2007)

[5] V. Boutueil, F. Leurent, «Les entreprises françaises face à l'automobilité et à la mobilité électrique : enjeux, mécanismes de décision et perspectives d'évolution », communication ATEC (2013)

[6] A. Appadurai, The Social Life of Things : Commodities in Cultural Perspective, Cambridge University Press (1986)

[7] P. Lejoux, «L'évolution des mobilités spatiales face aux impératifs du développement durable : quelle spécificité des stratégies d'adaptation des ménages résidant en ville intermédiaire ? », in M. Giroud, H. Mainet, C. Édouard, Les mobilités spatiales dans les villes intermédiaires. Territoires, pratiques, régulations, Presses Universitaires Blaise Pascal, Clermont-Ferrand, pp. 31-48 (2011)

[8] M. Pierre, «Script technique et prescriptions organisationnelles : utiliser un véhicule hybride rechargeable en milieu professionnel », in J. Cihuelo, C. Grandclément et A. Jobert, Sociologie des interfaces énergétiques (titre provisoire), éditions Lavoisier, Paris (2014)

[9] M. Dijk, R.J. Orsato, R. Kemp, “The emergence of an electric mobility trajectory”, Energy Policy, 52, pp. 135-145 (2012) 